Unfallchirurg $2021 \cdot 124: 173-174$

https://doi.org/10.1007/s00113-021-00968-x

Angenommen: 1. Februar 2021

(c) Springer Medizin Verlag $\mathrm{GmbH}$, ein Teil von Springer Nature 2021

\author{
H. Polzer ${ }^{1}$ S. Rammelt ${ }^{2}$ \\ ${ }^{1}$ Sektion Fuß- und Sprunggelenkchirurgie, Klinik für Allgemeine, Unfall- und Wiederherstellungschirurgie, \\ Klinikum der Universität München, LMU München, München, Deutschland \\ ${ }^{2}$ UniversitätsCentrum für Orthopädie, Unfall- und Plastische Chirurgie, Universitätsklinikum Carl Gustav \\ Carus an der TU Dresden, Dresden, Deutschland
}

\title{
Frakturen des Sprunggelenkes
}

Sehr geehrte Leserinnen und Leser!

Sprunggelenkfrakturen gehören aufgrund ihrer hohen Inzidenz zur täglichen unfallchirurgischen Routine [1, 2]. Allerdings sind die Behandlungsergebnisse, v. a. für Bi- und Trimalleolarfrakturen, häufig unbefriedigend [3]. Einige nichtbeeinflussbare Risikofaktoren für ein schlechtes Ergebnis wurden bereits identifiziert (hoher BodyMass-Index, fortgeschrittenes Patientenalter, Verletzung mehrerer Malleoli, Syndesmoseninstabilität) [4, 5]. Einige Faktoren können aber durch den Operateur positiv beeinflusst werden, wie beispielsweise die Qualität der Reposition, die Identifizierung und Behandlung von intraartikulären Begleitverletzungen, die anatomische und dauerhafte Wiederherstellung des distalen Tibiofibulargelenks sowie die Verminderung von Komplikationen durch die Wahl der geeigneten Operationstechnik [4, 6 , 7]. Lange Zeit wurden bei der Behandlung der Sprunggelenkfrakturen althergebrachte Behandlungsstrategien und Dogmen unkritisch übernommen. In den letzten Jahren wurden einige neue Therapieansätze zur Behandlung von Sprunggelenkfrakturen entwickelt. Im Rahmen dieses Themenheftes werden einige dieser aktuellen Entwicklungen dargestellt und diskutiert.

In vielen Diskussionen über moderne Entwicklungen in der Sprunggelenkschirurgie spielt die Möglichkeit der konservativen Therapie eine untergeordnete Rolle. Bei der operativen Therapie sind Komplikationen, wie beispielsweise Wundkomplikationen, nicht selten. Diese könnten durch eine konservative Therapie deutlich reduziert oder sogar ganz vermieden werden. Welche Frak- turen sind aber für eine konservative Behandlung geeignet, wie sollte diese durchgeführt werden, und wie sind die Ergebnisse? Dr. A. Baumann (Lancashire Teaching Hospitals NHS Trust, Manchester, England) et al. werden diesen Fragen nachgehen.

Die Empfehlung, ob eine Fraktur des posterioren Malleolus, des „VolkmannDreiecks" versorgt werden sollte, richtete sich jahrzehntelang ausschließlich nach der Größe des Fragments. Für Fragmente kleiner $25 \%$ der tibialen Gelenkfläche (in der Sagittalebene) wurde eine operative Versorgung bislang als nicht notwendig erachtet. Nur für Fragmente größer als $25 \%$ wurde eine Fixierung, häufig mittels perkutan in anteroposteriorer Richtung eingebrachter Schrauben nach indirekter Reposition, empfohlen. Dieses Konzept wird in den letzten Jahren zunehmend kritisch gesehen. Durch zahlreiche klinische und biomechanische Studien sowie den konsequenten Einsatz der CT bei der Beurteilung dieser Verletzungen haben sich inzwischen differenziertere Behandlungsalgorithmen herauskristallisiert. Prof. Dr. T. Mittlmeier (Abteilung für Unfall-, Hand- und Wiederherstellungschirurgie, Universität Rostock) et al. werden diese Empfehlungen kritisch hinterfragen.

Gerade bei (scheinbar) isolierten Frakturen des lateralen Malleolus kommt es immer wieder zu Verletzungen des Deltabandkomplexes. Diese Verletzungen sind wahrscheinlich deutlich häufiger als bislang angenommen. $\mathrm{Ob}$ sie aber relevant sind und ob, bzw. wann, sie versorgt werden sollten, wird kontrovers diskutiert. Prof. Dr. med. A. Barg (Universitätsklinikum Eppendorf, Hamburg) et al. werden anhand der aktuellen Literatur die Fragen beantworten: Wie können die 
Verletzungen des Deltabandkomplexes im Rahmen von Sprunggelenkfrakturen erkannt werden, und wie sollten sie behandelt werden?

Die Verletzungen des Syndesmosenkomplexes rücken bei der Behandlung der Sprunggelenkfrakturen immer mehr in den Fokus, da sie einen relevanten Faktor für das Ergebnis darstellen. Übersehene und unzureichend reponierte knöcherne Syndesmosenausrisse führen immer wieder zu Fehlstellungen und Instabilitäten der Knöchelgabel. Insbesondere die knöchernen Verletzungen der ventralen Syndesmose sind bislang in der Literatur noch wenig untersucht, erfahren aber durch verbesserte Diagnostik zunehmende Aufmerksamkeit. Die Arbeitsgruppe aus Dresden (Universitätsklinikum Carl Gustav Carus an der TU Dresden) wird die vorhandene Evidenz und aktuelle Überlegungen zur Behandlung dieser Verletzungen darstellen.

Immer mehr Menschen im höheren Alter erleiden Frakturen des Sprunggelenks. Dies liegt an der höheren Lebenserwartung und am höheren Aktivitätsniveau der Patienten. Die Frakturen beim älteren Patienten sind häufig komplexer, bedingt durch die reduzierte Knochenqualität. Zusätzlich können diese Patienten oft nur eingeschränkt eine Teilbelastung durchführen. Auch leiden sie an einem höheren Risiko für allgemeine und spezielle Operationsrisiken. Entsprechend sind bei der der Planung der Versorgung einige Besonderheiten zu beachten. Diese Aspekte werden Frau Prof. Dr. med. S. Ochman (Klinik für Unfall-, Hand und Wiederherstellungschirurgie, Universitätsklinikum Münster) et al. darstellen.

Die Nachbehandlung nach operativ versorgten Sprunggelenkfrakturen ist schon lange Gegenstand wissenschaftlicher Untersuchungen. Die erste prospektive randomisierte Studie zu diesem Thema wurde bereits vor 34 Jahren veröffentlicht. Seitdem sind insgesamt 18 randomisierte Untersuchungen veröffentlicht worden, die Vollbelastung mit Teil- oder Entlastung bzw. Mobilisation mit Immobilisation vergleichen. Unabhängig von den Ergebnissen dieser Studien wird in den allermeisten Fällen, nach althergebrachten Traditionen, eine 6-wöchige Teil- oder Entlastung durchgeführt, oftmals kombiniert mit einer Immobilisation. Das Team aus München (Klinik für Allgemeine-, Unfallund Wiederherstellungschirurgie, LMU München) wird einen Überblick über die vorhandenen Studien zur Nachbehandlung nach operativ versorgten Sprunggelenkfrakturen geben.

Obwohl Sprunggelenkfrakturen $\mathrm{zu}$ den häufigsten Brüchen überhaupt gehören, sind die Anzahl und Qualität der vorhandenen Evidenz eingeschränkt. Außerdem sind die vorhandenen Studien in den allermeisten Aspekten nicht vergleichbar. Hier sind beispielsweise $\mathrm{zu}$ nennen die durchgeführte Bildgebung (Röntgen/CT), die verwendeten Klassifikationen (AO-/Lauge-Hansen-Klassifikation), die Ein- und Ausschlusskriterien sowie die erhobenen Parameter und der Zeitpunkt deren Erhebung. Als Folge können aus der vorhandenen Evidenz oftmals keine eindeutigen Empfehlungen abgeleitet werden. Für die Zukunft wäre eine Vereinheitlichung dieser Aspekte sehr zu begrüßen. Dadurch könnten die Vergleichbarkeit und somit die Aussagekraft der durchgeführten Studien erheblich gesteigert werden.

Wir danken allen Autoren für die prägnante Darstellung der Themenbereiche und wünschen Ihnen eine interessante Lektüre zum Thema „Frakturen des oberen Sprunggelenks“.

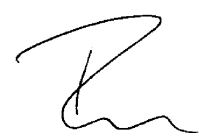

\section{H. Polzer}

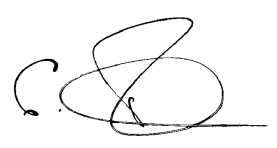

S. Rammelt

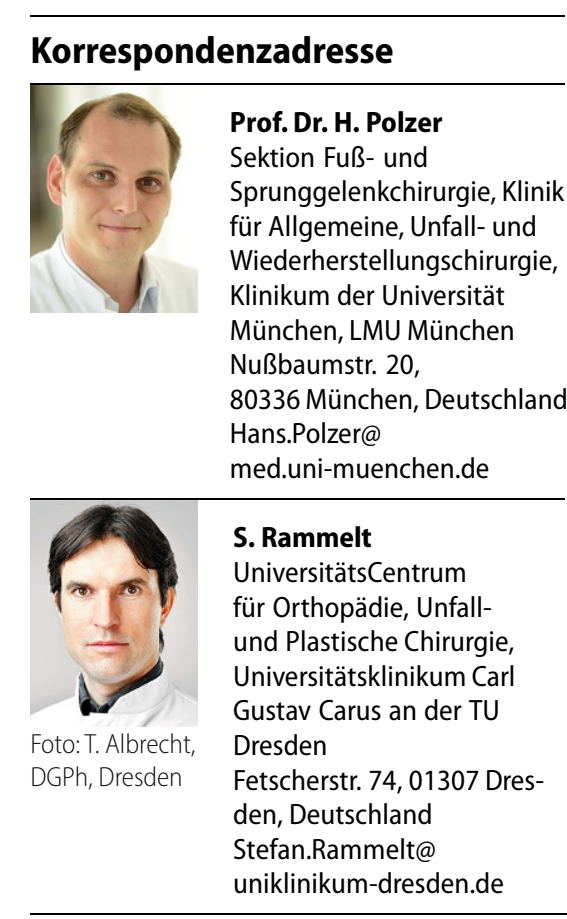

Interessenkonflikt. H. Polzer und S. Rammelt geben an, dass kein Interessenkonflikt besteht.

\section{Literatur}

1. Thordarson DB, Bains R, Shepherd LE (2001) The role of ankle arthroscopy on the surgical management of ankle fractures. Foot Ankle Int 22(2):123-125

2. Weimert S, Kuhn S, Rommens PM, Beutel ME, Reiner I (2019) Psychische Faktoren bei Sprunggelenkfrakturen. Unfallchirurg 122(6):464-468

3. Hong CC, Roy SP, Nashi N, Tan KJ (2013) Functional outcome and limitation of sporting activities after bimalleolar and trimalleolar ankle fractures. Foot Ankle Int 34(6):805-810

4. Kamin K, Notov D, Al-Sadi O, Kleber C, Rammelt S (2020) Versorgung der Sprunggelenkfraktur Standards, Tipps und Fallstricke. Unfallchirurg 123(1):43-56

5. Rammelt S, Manke E (2018) Syndesmosenverletzungen. Unfallchirurg 121(9):693-703

6. Braunstein M, Baumbach SF, Bocker W, Mutschler W, Polzer H (2016) Arthroskopisch assistierte Frakturversorgung am oberen Sprunggelenk. Unfallchirurg 119(2):92-98

7. Baumbach SF, Bocker W, Polzer H (2020) Arthroskopisch assistierte Frakturversorgung und offene Reposition des posterioren Malleolus - Neue Strategien zur Versorgung komplexer Sprunggelenksfrakturen. Unfallchirurg 123(4):330-338 